\title{
Assessment of the Perceived Quality of Life of Non insulin Dependent Diabetic patients attending the Diabetes Clinic in Kenyatta National Hospital
}

\author{
Genga E.K, Otieno C.F, Ogola E.N Maritim M.C \\ Department of Clinical Therapeutics and Medicine, University of Nairobi.
}

BACKGROUND: Diabetes Mellitus is a common and demanding health related problem that has a wide effect on every day's life of the patients. It can have a profound effect on quality of life in terms of social and psychological well-being as well as physical ill-health. It is one of the most psychologically demanding of the chronic diseases; with psychosocial factors pertinent to nearly every aspect of the disease and its treatment.

OBJECTIVE: To Assess the perceived Health-related quality of life of diabetic patients not on insulin therapy using the WHOQoL-Bref (World Health Organization Quality of Life - Brief).

STUDY DESIGN: This was a cross-sectional study.

STUDY SITE: The study was conducted on patients attending the Diabetic clinic at Kenyatta National Hospital.

RESULTS: Study recruited 139 patients with type 2 diabetes not on insulin therapy. The study population was predominantly female (61\%), majority were 40-60yrs, having had diabetes for less than 5yrs, $75 \%$ having more than one complication. Most $(75 \%)$ of the study participants were poorly controlled with HbA1C mean score of 8.04\% .Majority of the study participants( $84 \%$ ) achieved a good score on the HRQoL scale using the WHOQoL-Bref tool. The determinants of HRQoL in our study were: age of study participants, duration of diabetes, presence of complications and income related factors. Age of the study subjects had significant association only in the social domain of HRQoL with a p-value of 0.037. Level of income had a significant association with overall HRQoL score (p-value of 0.029), psychological domain ( $\mathrm{p}$ value of 0.023 ) and in the social domain ( $\mathrm{p}$-value of 0.029 ). Health care financing was significantly associated with psychological domain (p-value 0.006) and environmental domain (p-value 0f 0.04) and overall score (p-value 0.011). There was an association between employment status and HRQoL. Having a job improved the scores in physical domain ( $\mathrm{p}-$ value of 0.013) and social domain ( $\mathrm{p}$ value of 0.020). Duration with diabetes had significant association with physical domain where the $\mathrm{p}$ value was 0.007 . The HRQoL of the study subjects was associated significantly with the number of complications. Indeed the association of complications with the HRQoL involved physical domain (p-value of $<0.0001$ ) and psychological domain (p-value of 0.041 ) which directly impacted on the overall total score ( $\mathrm{p}$ value of 0.041 ).

CONCLUSION: The results of this study show that diabetes affected HRQoL of our study participants. There is a need for interventions programs to improve glycemic control and inclusion of HRQoL assessment as part of patients on follow up. Age and duration of disease are not modifiable but complications can be reduced by better health care initiaves. Income-related factors can be modifiable through poverty alleviation and pooled health care financing.

\section{INTRODUCTION}

Diabetes Mellitus is a common and demanding health related problem that has a wide effect on every day's life of the patients ${ }^{1}$. Diabetes mellitus is a chronic illness and the most common endocrine disease, although the disease is prevalent worldwide, there is significant difference frequency among countries ${ }^{2,3}$ and the major public health problem for both developed and developing countries ${ }^{4}$. The increasing number of individual with type 2 diabetes indicates a global epidemic. Prevalence of the disease was estimated to be $2.8 \%$ in 2000 and is predicted to increase to $4.4 \%$ by the year $2030^{5}$. The prevalence of diabetes is rising in Africa and the Third world countries due to adaptation of the western lifestyle and $\operatorname{diet}^{6}{ }^{6}$ In Africa, the traditional rural communities still have low prevalence of 1-2\% (except in specific high risk groups) while 1-13\% or more adults in urban communities have diabetes with Kenya having an estimated prevalence of between $3.3 \%{ }^{7}$. Diabetes can have a profound effect on quality of life in terms of social and psychological well-being as well as physical ill-health. It is one of the most psychologically demanding of the chronic diseases; with psychosocial factors pertinent to nearly every aspect of the disease and its treatment ${ }^{5}$. In a longitudinal study the psychosocial impact 
of diabetes was found to be one of the five strongest predictors of mortality in diabetic patients, stronger than many clinical and physiological variables ${ }^{8}$.

The health-related quality of life (HRQoL) deficits reported by people with diabetes are generally attributed to the disease itself, its restrictive treatment regimens including diet, and its associated co morbidities. Living with diabetes has financial, social and psychological handicaps though the patient is encouraged to lead a "normal life". The treatment is demanding and often complex. The patient is expected to bear much of the responsibility for making decisions which affect his/her health, both in the short- and long-term. HRQoL will help evaluate the efficacy, cost effectiveness, and net benefits of current programs and interventions. There is a paucity of data in developing countries with deteriorating economic environment compounded with social and cultural influences and its impact on patients and care of disease.

Measuring QoL changes usually involves soliciting peoples' self-reported feelings, behaviors and attitudes through interviewing or evaluating responses to questionnaires. A variety of instruments has been used to measure HRQoL in diabetes. Examples include WHO-QoL BREF tool, SF-36 tool, QWB-SA (Quality of Well-Being Questionnaire), EuroQoL (European Quality of Life) or EQ-5D (Euro-QoL 5-Dimensions), DQLCTQ-R (Diabetes Quality of Life Clinical Trials Questionnaire Revised), ADDQoL (Audit of Diabetes Dependent QoL) instrument etc we chose WHOQoL-BREF because it has good to excellent psychometric properties of reliability and validity. It has been validated in people with type 2 diabetes ${ }^{9}$.

\section{PATIENT METHODS:}

The study was performed in the diabetic clinic at the Kenyatta National Hospital which serves approximately 400 patients per month. This the biggest referral hospital in Kenya based in Nairobi the capital City of Kenya.

\section{Patients}

The sample population will be obtained from the Diabetic clinic at the KNH which runs from Monday to Friday. The main clinic is on Friday which sees on average 40 patients per session whilst the mini clinic which runs from Monday to Thursday sees on average 20 patients per day. Consecutive sampling was used until minimum sample of 129 , in total 139 patients were recruited based on the following:

\section{Inclusion criteria:}

[1.] DM patient diagnosed via WHO criteria aged between 18yrs and 70yrs

[2.] Known diabetic for 1year

[3.] Stable disease-no admissions in past 3months

[4.] Informed consent

\section{Exclusion criteria}

[1.] History of cognitive impairment

[2.] History of substance abuse

[3.] History of concomitant disease that could affect the nervous system or independently affect the quality of life.

[4.] Gestational diabetes

[5.] Diabetic patients on insulin

\section{STUDY INSTRUMENT}

Instrument consisted of social demographic Questionnaire and WHOQoL-Bref Questionnaire. The WHO-QoL tool has 4domains. The four domain scores denote an individual's perception of quality of life in each particular domain which are: physical, psychological, social and environment, through a set of 26 items that can be self-administered. Responses to the questions use a 5-point Likert scale, inquiring 'how much', 'how satisfied' or 'how completely' the respondent felt in relation to the domain being investigated.

The mean score of items within each domain is used to calculate the domain score. Mean scores are then multiplied by 4 in order to make domain scores comparable with the scores used in the WHOQoL-100 (Higher scores denote higher quality of life). When more than $20 \%$ of data is missing from assessment, the assessment was discarded. Where an item was missing, the mean of other items in the domain is substituted but when more than two items were missing from the domain, the domain score was not calculated (with the exception of domain 3, where the domain should only be calculated if $<1$ item is missing). Thereafter, raw scale scores were deduced and were finally transformed to a scale of $0-100$. The WHOQoL-Bref Questionnaire was either self answered or administered by the investigating team. 


\section{RESULTS}

The study population was aged mainly between $40-60$ years, with a mean of 56.37 years, most were females $61.3 \%$ ( $n=84) .57 .6 \%$ of the sample population were married with the remainder classified as single (widowed/ divorced/ separated/ single). The population was a well-educated group with up to $90 \%$ having received a minimum of primary school education though majority of study patients needed help in filling the questionnaire. Up to $60 \%$ of the sample population had some employment whether part or full time with $58.4 \%$ having a combined income of more than 50,000 a year. $87 \%$ of the population was personally responsible for their house meaning either owned the house or paid rent. Another $80 \%$ were able to pay for their own healthcare. Majority of the study patients (52.5\%) had diabetes for less than 5yrs and at least $75 \%$ had 3 or more complications $.80 \%$ were rated as overweight and obese as per WHO standards with a mean BMI of $29.1 \mathrm{~kg} / \mathrm{m}^{2}$, The mean $\mathrm{HbA1C}$ was $8.04 \%$ this translated to $24.5 \%$ meeting the recommended target level of $<7 \% .52 .5 \%$ of the patients had been diabetic for less than 5years, with up to $57.6 \%$ of the patients with more than three complications. Neuropathy (41\%) affected the most of the patients. 35-40\% of our study patients rated their life as good and was satisfied with their health but when it came to the overall QoL score $84 \%$ scored as good QoL. All the domains were affected with physical and psychological most affected. There was association between age and HRQoL in the social domain where the older the patient the worse the score with a p-value of 0.037.The correlation between the HRQoL and level of income was found to be significant especially in the psychological domain ( $p$-value of 0.023) and in the social domain ( $p$-value of 0.029) There was an association between the overall HRQoL and mode of health care funding with patients with private insurance scoring highest, then self paying, government assistance and last patients on family support with a p-value of 0.011 . The domains affected were psychological ( $p$-value 0.006) and environmental ( $p$-value of 0.04 ). There was a significant association between employment status and HRQoL. Having a job improved the scores in physical (p-value of 0.013) and social domains ( $\mathrm{p}$-value of 0.020 ). There was a significant association between HRQoL and duration of diabetes as evident in the physical domain where the $\mathrm{p}$ value was 0.007 . Also a significant association between HRQoL and duration of diabetes was seen as evidenced in the physical domain where the p-value was 0.007. There was a significant association between HRQoL and number of complications where as the number of complications increased the worse the HRQoL score which was evidenced in physical domain ( $\mathrm{p}$-value of <0.0001) and psychological domain ( $\mathrm{p}$-value of 0.041 ) which directly impacted on the overall total score ( $\mathrm{p}$-value of 0.041 )

TABLE 1- Socio-demographic characteristics of the study population

\begin{tabular}{llc}
\hline Characteristic & Category & \% \\
\hline Gender & Male & $38.7 \%$ \\
& Female & $61.3 \%$ \\
\hline Level of education & None & $8.7 \%$ \\
& Primary & $44.9 \%$ \\
& High School & $34.1 \%$ \\
& College & $12.3 \%$ \\
& & \\
\hline Marital status & Single & $42.4 \%$ \\
& Married & $57.6 \%$ \\
& & \\
\hline Housing & not personally responsible & $12.9 \%$ \\
& personally responsible & $87.1 \%$ \\
& & \\
\hline Employment & Unemployed & $39.9 \%$ \\
& Employed & $60.1 \%$ \\
& & \\
\hline Total combined family & Less than ksh5,000 & $15.1 \%$ \\
income for the past 12 & Ksh5,000 - Ksh19,999 & $9.4 \%$ \\
months & Ksh20,000 - Ksh49,999 & $17.0 \%$ \\
& Ksh50,000 - Ksh99,999 & $15.1 \%$ \\
& Ksh100,000 - Ksh149,999 & $12.3 \%$ \\
& More than Ksh150,000 & $31.1 \%$ \\
& & \\
& &
\end{tabular}


Table 2 HRQoL domain scores of the study subjects

\begin{tabular}{lccccccc}
\hline & N & Mean & Median & \% score & $\begin{array}{c}\text { Minimu } \\
\text { m }\end{array}$ & $\begin{array}{c}\text { Maximum } \\
\text { Standard } \\
\text { Deviation }\end{array}$ \\
\hline DOM1: Physical Health & 139 & 14.01 & 14.86 & $70.1 \%$ & 7.00 & 20.00 & 3.23 \\
\hline DOM2: Psychological & 139 & 14.58 & 14.67 & $72.9 \%$ & 6.40 & 20.00 & 2.76 \\
\hline DOM3: Social relationships & 139 & 14.75 & 14.67 & $73.8 \%$ & 8.00 & 20.00 & 3.09 \\
\hline DOM4: Environmental & 139 & 13.66 & 13.50 & $68.3 \%$ & 6.50 & 20.00 \\
\hline Total Domain Scores & 139 & 56.94 & 57.90 & $72.4 \%$ & 30.76 & 78.86 \\
\hline
\end{tabular}

Table 3 overall HRQoL scores of the study subject

\begin{tabular}{|c|c|c|c|}
\hline HRQoL scores & $\begin{array}{l}\text { Categories of HRQoL } \\
\text { scores }\end{array}$ & $\mathbf{N}$ & $\%$ \\
\hline \multirow{3}{*}{$\begin{array}{l}\text { Total Scores } \\
\text { Maximum } 100\end{array}$} & $<=40($ poor $)$ & 1 & $0.7 \%$ \\
\hline & 41-60(fair) & 21 & $15.2 \%$ \\
\hline & $>60($ good $)$ & 117 & $84.1 \%$ \\
\hline
\end{tabular}

Table 4 Health Satisfaction and Self rating of HRQoL of the study subjects

\begin{tabular}{llll} 
Self-Rating of quality of life & $\%$ & Health satisfaction & $\%$ \\
\hline Very poor & $6.5 \%$ & Very dissatisfied & $7.9 \%$ \\
Poor & $15.8 \%$ & Dissatisfied & $30.2 \%$ \\
Neither poor nor good & $37.4 \%$ & Neither dissatisfied nor satisfied & $25.9 \%$ \\
Good & $33.8 \%$ & Satisfied & $34.5 \%$ \\
Very good & $6.5 \%$ & Very satisfied & $1.4 \%$ \\
\hline
\end{tabular}

Table 5 Association between HRQoL domains and determinants

\begin{tabular}{lllccc}
\hline \multicolumn{1}{l}{ variable } & $\begin{array}{l}\text { total } \\
\text { Score }\end{array}$ & physical & psychological & Social & environmental \\
\hline Age & 0.609 & 0.470 & 0.937 & $\mathbf{0 . 0 3 7}$ & 0.136 \\
\hline gender & 0.955 & 0.094 & 0.825 & 0.579 & 0.308 \\
\hline Marital status & 0.712 & 0.405 & 0.309 & 0.126 & 0.803 \\
\hline Income & $\mathbf{0 . 0 2 9} *$ & 0.507 & $\mathbf{0 . 0 2 3} *$ & $\mathbf{0 . 0 2 9} *$ & 0.153 \\
\hline Education level & 0.753 & 0.358 & 0.826 & 0.390 & 0.701 \\
\hline Health care funding & $\mathbf{0 . 0 1 1} *$ & 0.082 & $\mathbf{0 . 0 0 6}$ & 0.138 & $\mathbf{0 . 0 4 0}$ \\
\hline employment & 0.078 & $\mathbf{0 . 0 1 3} *$ & 0.366 & $\mathbf{0 . 0 2 0}$ & 0.941 \\
\hline DM Duration & 0.300 & $\mathbf{0 . 0 0 7} *$ & 0.218 & 0.763 & 0.281 \\
\hline Complications & $\mathbf{0 . 0 4 1} *$ & $\mathbf{< . 0 0 0 1} *$ & $\mathbf{0 . 0 4 1} *$ & 0.876 & 0.103 \\
\hline BMI & 0.574 & 0.573 & 0.235 & 0.806 & 0.810 \\
\hline HbA1C & 0.515 & 0.772 & 0.800 & 0.304 & 0.136 \\
\hline
\end{tabular}

\section{DISCUSSION}

This study sought to determine the overall quality of life in ambulatory patients with type 2 diabetes on oral hypoglycemic agents and made important observations and findings. Of the consented interviewed 139 subjects majority were female $(61.3 \%)$. This is consistent with other studies from Nigeria, India and Thailand ${ }^{10-}$ ${ }^{12}$.Probable reasons for the female predominance were likely because this was a hospital-based study where access to care is selected by affordability, referral, proximity and severity of illness. The main age group of the 
study population was between 40 and 60 years with a mean of 56.37 years. These ages are important because that is an age-group bracket of active economic activity for both self-care and family. This was confirmed as $60 \%$ the study participants had a source of employment. The quality of life of the study subjects was good with approximately $84 \%$ scored above fair on the overall quality of life scale. This is comparable with other studies done in Nigeria and Kuwait ${ }^{10,13}$. These two studies were cross-sectional studies using similar tool used in this study. This contrasts with studies done in South Africa where diabetes was found to have negative impact on HRQoL of majority of the study subjects though important to note they used a different tool and also had a smaller sample size ${ }^{14}$. A minority $(40 \%)$ of the subjects rated their health as good and equally low proportion $(35 \%)$ were satisfied with their health status. This is disturbing as we know that diabetes is a chronic disease and can be a difficult condition to live with for many patients. The demand of self-care can be burdensome, frustrating and overwhelming both in terms of commitment to self-care and resource consumption (medication, monitoring and attending hospital). All the domains of HRQoL were affected with social and physical domains most affected.

Poor quality of life was significantly associated with age, level of income and health care financing, employment status, duration of diabetes and its complications. Age of the participants emerged as a significant association with quality of life, on the social domain and not in the three other domains. A plausible interpretation is that interactions of aging-related disabilities with complications of diabetes seem to have influence on the social domain of the study patients. The peak age of type 2 diabetes in this study is a relatively younger age group (35 -55 years) in this country as compared to the developed world of (55-75 years) ${ }^{15}$.Aging overlaps with duration of diabetes in patients, and this is compounded by complication loading (which is a factor of genes, quality of care and metabolic control). It is therefore not surprising that duration of diabetes, number of complications per person were significantly associated with quality of life of the study participants. This is similar to other studies done in Nigeria, Thailand, South Africa and by the Zodiac group ${ }^{10,12,14,16}$.

Diabetes as a chronic disease is demanding in both self-care commitment and resource consumption (medication, monitoring and attending hospital). It is therefore imperative that ability to meet these requirements may affect one's own quality of life. Majority of the study population are in the bracket of active economic activity for both self-care and family. It is known that diabetes on its own can affect income generation directly and indirectly through complications e.g. neuropathy, retinopathy etc. thus quality of life studies are important in the context of one's personal well-being and the ability to work and earn a living. This study found that employment status, ability to purchase own health care and high annual income levels were significantly associated with physical, psychological and social domains of quality of life as shown in tables 5. Studies in Nigeria, South Africa, India and Thailand have made similar observations though the context of health care provision of patients, organizations of diabetes care and financing may vary in environments within which these studies were conducted ${ }^{10,11,12,14}$.

Just about half of the study patients $(52.5 \%)$ had diabetes for less than $5 y r s$ and at least 3 out of 4 study subjects had 3 or more complications. The most common complication was neuropathy with $41 \%$ of the study population. This finding is similar in other studies that have found neuropathy as the most common complication with Jacobson et al. ${ }^{17}$ reported that $48.8 \%$ of their subjects had neuropathy; Mayou and Bryant found $20 \%$ with neuropathy ${ }^{18}$; and Weinberger et al reported $24 \%$ with neuropathy ${ }^{19}$. Duration of diabetes and complication loading affected the physical, social and psychological domains which compares to most of the studies done on quality of life in Nigeria ${ }^{10}$, Kuwait ${ }^{13}$, Sweden ${ }^{14}$, CODE-study ${ }^{20}$ and South Africa ${ }^{21}$. This study found that complications such as neuropathy (40\%) and retinopathy affected physical and psychological domains. It also found that others like erectile dysfunction adversely affected the psychological domain. New onset erectile dysfunction has been shown to be a marker of complications (macrovascular and neuropathy) and worsening quality of life of diabetic patients ${ }^{22}$. Duration of diabetes, complications (retinopathy, neuropathy, erectile dysfunction, diabetic foot etc.) as well as advancing in age also contributed as Increase affected their social lives, income generating activities and increase amount spent on diabetes health care. This was reflected in poor scores in physical and social domains. Jacobson et al studied both type 1 and 2 diabetes and found that quality of life decreased in relation to the number of complications of their study patients ${ }^{17}$. Rubin et al noted that the presence of co-morbid conditions and unfavorable socio-economic characteristics can further interact with the severity of diabetes and its complications to strongly influence different domains of quality of $\operatorname{life}^{23}$.This has been partially demonstrated in this study.

Glycemic control in this group was poor as only 1 in 4 of the study subjects attained the target HbA1C level of $<7 \%$ and the mean was $8.04 \%$. This is comparable to an earlier study done in this clinic on glycemic control by Otieno $\mathrm{C}$ et $\mathrm{al}^{24}$. The poor metabolic control may be explained by multiple probable reasons that include irregularity of testing due to various reasons including adequate financing for diabetic healthcare, lack of adequate knowledge by patients on diabetes self-care, medication and relevance of regular testing of $\mathrm{HbA} 1 \mathrm{C}$ 
(actually majority (90\%) had no idea what $\mathrm{HbA1C}$ was). The quality of glycemic control by HbAlc did not influence the HRQoL and its domains in this study. ZODIAC-2 study also made a similar finding in their study of quality of life of patients with type 2 diabetes ${ }^{16}$. The serial blood sugars may have been a better tool to use because of the symptoms of both hyperglycemia (polyuria, poor vision etc.) and hypoglycemia (loss of consciousness etc.) would have had a larger impact on the quality of life especially over a two week duration rather than $\mathrm{HbAlC}$ which is more of a historical assessment. The absence of hyperglycemic symptoms in relation to the level of HbA1c as shown by the ZODIAC- $2^{16}$ and QUED $^{22}$ study groups may have some explanation. These two studies found that poor glycemic control led to more hyperglycemic symptoms which impacted on the quality of life thus underscoring the importance of symptoms in determination of one's quality of life. Similar results were seen in a study done in Thailand though they used random blood sugar instead of $\mathrm{HbA1C}$ used in our study ${ }^{12}$. This contrasts with study done in South Africa where they showed that glycemic control it does impact the quality of life though they used a different tool to measure the quality of life ${ }^{14}$.

However, marital status, gender and level of education of our study participants were not significantly associated with their quality of life. Marital status of study participants did not affect quality of life. This is surprising because one would expect better psychosocial support in the married ones as seen in studies from India and Sweden ${ }^{11,20}$. Possible explanations are gender bias may have skewed the results and good support from family and extended family may have contributed but still difficult to conclude. Level of education had no impact on quality of life which is surprising because diabetes treatment is demanding and often complex, the patient is expected to bear much of the responsibility for making decisions which effect his/her health, both in the short- and long-term. Plausible explanation is that majority (90\%) had received minimum primary education. This contrasts with other study done in India though it used a different tool and had a larger sample $\operatorname{size}^{11}$.

\section{CONCLUSIONS AND RECOMMENDATIONS}

There is reduced quality of life in our patients with diabetes. This is compounded by a small percentage of the study subjects (35-40\%) satisfied with their health. There was poor metabolic control as evidenced by large number having multiple complications and poor $\mathrm{HbA1C}$ despite having had diabetes for a short duration of time. The determinants of HRQoL in the study patients in our study were: age of study participants, duration of diabetes, presence of complications and income related factors- employment status, amount of income, mode of health care financing. Factors that were not associated with HRQoL in our diabetic population were: gender, marital status, level of education, BMI and HbA1C. This study was able to show that being an older age, having had diabetes for a longer duration with multiple complications and inability to afford health care are likely to have poor HRQoL scores.

Issues raised included: poor Glycemic control, overweight and obesity, complication profile in our patients despite a large majority of our patients having been diagnosed with diabetes less than 5 years ago. Other issues raised in our study were socio-economic support for our patients as this impacted heavily on the overall quality of life and all the domains. In conclusion this study has added information about diabetes care in Kenya and a follow up study on the same population maybe in the future to see if the quality of life has changed, if interventions have been implemented to see the effect and do comparative study in the various provinces and see its relation to our study.

\section{Limitations:}

1. Recall bias

2. Response bias - it was administered by the investigator and some of the questions were too personal this may have led to a response bias

3. Life events and psychiatric disorders in previous time beyond 2 weeks which could affect subjective QOL could not be ruled out

4. Hospital based study: Lack of generalisability to the rest of the diabetic population in Kenya

5. Lack of insulin arm amongst the study participants to act as a comparison group.

\section{REFERENCES}

[1] Faro B. (1999). The effect of diabetes on adolescent quality of life. Pedi Nurs. 25 (247-254).

[2] American Diabetes Association. Clinical practice Recommendations. (2002) Diabetes care. 25 (suppl.1)

[3] Qari F.A. (2005). Glycemic and Good Target Control among Diabetics at a university and Erfan private hospital. Turkish journal of Endocrinology and Metabolism. (2), 49-54.

[4] K. Inoue, M. Masatoshi Matsumoto, Y. kobayashi, (2007). The combination of fasting plasma glucose and glycosylated hemoglobin predicts type 2 diabetes in Japanese workers. Diab. Res. Clin.Pract. 1-8.

[5] Cox WM, Blount JP, Crowe PA, Singh SP. (1996). Diabetic patients' alcohol use and quality of life: Relationships with prescribed treatment compliance among older males. Clin Exp; 20: Res (20), 327-331.

[6] Sobngwi E,, Maurvais-Jarvis, F, Mbanya, J.C. (2007) Diabetes in Africa: epidemiology and clinical specifities.

[7] Kenya Diabetes Study Group 2011.

[8] Davis WK, Hess GE, Van Harrison R, Hiss RG. (1998) Psychosocial correlates of survival in diabetes. Diabetes Care; 11: 538-545. 
[9] Skevington, S. M, Lotfy, M, \& O'Connell, K. A. (2004). The World Health Organization's WHOQOL-BREF quality of life assessment: psychometric properties and results of the international field trial. A Report from the WHOQOL Group. Quality of Life Research; 13 (2), 299- 310.

[10] B A Issa, O Baiyewu. (2006) Quality of life of patients with diabetes in a Nigerian Teaching Hospital. Hong Kong J Psychiatry, 16(1) $: 27-33$

[11] Gautam Y, Sharma AK, Agarwal AK, Bhatna-gar MK, Trehan RR.(2009). A cross-sectional study of QOL of diabetic patients at tertiary care hospitals in Delhi. Indian J Community Med 34(4):346-50.

[12] Natchaporn P, Wisit C. (2011). Self-care activities and quality of life among Type-2 diabetic patients. J Sci Technol MSU 30(1): $74-$ 83.

[13] W Bani-Issa. (2011). Evaluation of the health-related quality of life of Emirati people with diabetes: integration of socio-demographic and disease-related variables United Arab Emirates East Mediterr Health J 17:825-30

[14] Katzenellenbogen, Leanne. (Stellenbosch: University of Stellenbosch University, 2008-12). Assessment of perceived impact of diabetes on quality of life in group of South African diabetic patients.

[15] WHO- "Diabetes Action Now" booklet 2011

[16] Kleefstra N, Ubink-Veltmaat LJ, Houweling ST, Groenier KH, Meyboom-de Jong B, Bilo HJ.(2005) Cross-sectional relationship between glycaemic control, hyperglycaemic symptoms and quality of life in type 2 diabetes (ZODIAC-2). Neth J Med. Jun;63(6):21521.

[17] Jacobson A M. (2004). Impact of improved Glycemic control on QOL in patients with diabetes. Endocrin Pract 10:502-08

[18] Weinberger M, Kirkman MS, Samsa GP, Cowper PA, Shortliffe EA, Simel DL, Feussner JR. (1994). The relationship between glycaemic control and health-related quality of life in patients with non-insulin-dependent diabetes mellitus. Med Care 32 (12):117381

[19] Mayour R, Bryant B, Turner R. (1990). Quality of life of non-insulin dependent diabetics and a comparison with insulin dependent diabetic J Pyschosom Res 34:1-11.

[20] Wredling R, Stalhammar J, Adamson U, Berne C, Larsson Y, Ostman J.(1995) Well-being and treatment satisfaction in adults with diabetes: a Swedish population-based study. Qual Life Res 4:515-522

[21] Koopmanschap MA (2002). (On behalf of theCode-2 advisory board): Coping with type 2 diabetes: quality of life. Diabetologia 45(7): S5-S12.

[22] De Berardis G, Pellegrini F, Franciosi M, Belfiglio M, Di Nardo B, Greenfield S et al. (2005). Longitudinal assessment of quality of life in patients with type 2 diabetes and self-reported erectile dysfunction. QuED (Quality of Care and Outcomes in Type 2 Diabetes) Study Group. Diabetes Care, 28(11):2637- 2643.

[23] Rubin RR, Peyrot M. Quality of life and diabetes.(1999). Diabetes Metab Res Rev 15 (3): 205-18.

[24] Otieno CF, Kariuki M, Nganga L. Quality of glycaemic control in ambulatory diabetics at the outpatient clinic of at Kenyatta National Hospital, Nairobi. East Afr Med J. 2003; 80:406-10. 